\title{
Editorial Medical Journal of Hospital General de México
}

\author{
Octavio Amancio-Chassin ${ }^{1 *}$, Mercedes Hernández-G. ${ }^{2}$, and Eduardo E. Montalvo-Javé ${ }^{3}$ \\ ${ }^{1}$ Servicio de Farmacología Clínica; ${ }^{2}$ Servicio de Patología; ${ }^{3}$ Servicio de Cirugía General, Clínica de Cirugía de Hígado, Páncreas y Vesícula Biliar. \\ Hospital General de México “Dr. Eduardo Liceaga”, Mexico City, Mexico
}

In science, researchers have always managed to share any acquired knowledge by means of a strict methodology, observational or experimental designs, which are specified in research protocols previously authorised by Research Ethics Committees and in some cases by the biosafety teams of scientific journals. The Medical Society of the General Hospital of Mexico, since its establishment, has taken an active role in disseminating knowledge acquired by physicians and health professionals, who conduct research studies at the services and units of Dr. Eduardo Liceaga's General Hospital of Mexico (Hospital General de México Dr. Eduardo Liceaga), aiming to identify new diagnostic methods or treatment for patients.

In this sense, Dr. Ignacio Chávez ${ }^{1}$, founding member of the Medical Society, created the Medical Journal (Revista Médica) of the General Hospital of Mexico, with the goal of publishing original articles, case reports and peer reviews that were disseminated among different medical activities held at the hospital, by the members of the Medical Society of the General Hospital of Mexico. The journal, during its 81 years of life, has evolved, by incorporating other sections, which make room for discussions ranging from health problems to the bioethical aspects of medicine (medical arbitration cases, medical education topics or briefing notes). The purpose has always been the dissemination of experiences of the health professionals, who are members of the Medical Society of the General Hospital of Mexico; as well as of different health or educational institutions on a nationwide level.

Throughout its history, different editorial committees have always endeavoured to take the medical journal to a higher level of pre-eminence, which allows for a better index ranking. Currently, the journal is indexed in ARTEMISA, LILACS, IMBIOMED, PERIÓDICA UNAM and ULRICH. However, the medical journal should be indexed to new platforms such as PubMed, MEDLINE, Scopus, etc., so that it has greater academic exposure. This target will only be possible if we can count on the entire support of the scientific community, the Medical Society of the General Hospital of Mexico, our Dr. Eduardo Liceaga's General Hospital of Mexico, and the group of reviewers who support us by reviewing different articles and manuscripts, which are submitted for publishing in our journal.

In October this year, our Editor-in-Chief Dr. Rafael Gutiérrez Vega resigned from his post, giving rise to a restructuring of the Editorial Committee. After an evaluation, the Editorial Committee of the journal and the Medical Society of the General Hospital of Mexico appointed Dr. Octavio Amancio Chassin, as editor-in-chief and the following doctors remained as associate editors: Mercedes Hernández and Eduardo E. Montalvo Javé.

\section{Correspondence:}

*Octavio Amancio-Chassin

E-mail: octaviorev28@gmail.com

0185-1063/@ 2019 Sociedad Médica del Hospital General de Mexico. Published by Permanyer. This is an open access article under the CC BY-
Date of reception: 05-12-2019

Date of acceptance: 17-12-2019

DOI: 10.24875/HGMX.M20000036
Available online: 17-01-2020 Rev Med Hosp Gen Mex. 2020;83(1):3-4 www.hospitalgeneral.mx
article under the CC BY- 
We would like to thank, on behalf of the Editorial Committee of the journal and the General Hospital of Mexico, Dr. Rafael Gutiérrez Vega, for his outstanding service as Editor-in-Chief of the journal in the years 2014-2019, a time of growth and development of the journal. He was the main force behind the publication of the English version of our journal. Issue \#74, of October-December 2014, was the first issue of the journal published in English. Furthermore, he summoned researchers from different services and units of the General Hospital of Mexico, as well as other institutions to publish in our Medical Journal, always on a regular basis and with suitable and rigorous content. We wish Dr. Gutiérrez Vega, our friend and fellow, greatest success in all his endeavours.

The Editorial Committee wishes to keep and improve the quality of the content of our publications, with a frequency and timing in accordance with the applicable quarter and year, by streamlining administrative and logistic processes for the review, acceptance and publication of different articles or manuscripts submitted by prominent physicians of various medical specialties within and outside Dr. Eduardo Liceaga's General
Hospital of Mexico. The Journal will have online versions in English and Spanish, so that direct PDF downloads are the most dynamic option for accessing the content of our publications. Arrangements will be made with the company in charge of the administration of the website of the Medical Journal of the General Hospital of Mexico, so that its platform has a more user-friendly interface, aimed at shortening review times of the articles and manuscripts for a quicker response time and more streamlined internal processing. With these changes, we plan to begin 2020 with a more consolidated image of our journal and committed to continuously improve and increase its academic impact.

We take the opportunity to invite the national and international scientific community from different health institutions, as well as educational institutions to submit their original, unpublished research or academic manuscripts, peer reviews, case reports, medical arbitration cases, medical education topics or briefing notes.

\section{References}

1. Díaz-de Kury M, Viesca-Treviño C. Historia del Hospital General de México 1905-1910. Hospital General de México. Ciudad de México, México, 1994. 